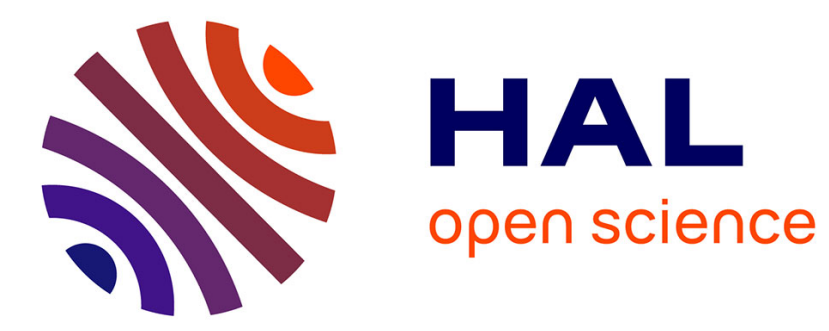

\title{
Can a sinking metallic diapir generate a dynamo?
}

\author{
Julien Monteux, Nathanaël Schaeffer, Hagay Amit, Philippe Cardin
}

\section{To cite this version:}

Julien Monteux, Nathanaël Schaeffer, Hagay Amit, Philippe Cardin. Can a sinking metallic diapir generate a dynamo?. Journal of Geophysical Research. Planets, 2012, 117 (E10), pp.E10005. 10.1029/2012JE004075 . hal-01636052

\section{HAL Id: hal-01636052 \\ https://hal.uca.fr/hal-01636052}

Submitted on 16 Nov 2017

HAL is a multi-disciplinary open access archive for the deposit and dissemination of scientific research documents, whether they are published or not. The documents may come from teaching and research institutions in France or abroad, or from public or private research centers.
L'archive ouverte pluridisciplinaire HAL, est destinée au dépôt et à la diffusion de documents scientifiques de niveau recherche, publiés ou non, émanant des établissements d'enseignement et de recherche français ou étrangers, des laboratoires publics ou privés. 


\title{
Can a sinking metallic diapir generate a dynamo?
}

\author{
Julien Monteux, ${ }^{1}$ Nathanaël Schaeffer, ${ }^{2}$ Hagay Amit, ${ }^{1}$ and Philippe Cardin ${ }^{2}$ \\ Received 5 March 2012; revised 21 August 2012; accepted 21 August 2012; published 10 October 2012.
}

[1] Metallic diapirs may have strongly contributed to core formations during the first million years of planetary evolutions. The aim of this study is to determine whether the dynamics induced by the diapir sinking can drive a dynamo and to characterize the required conditions on the size of the diapir, the mantle viscosity and the planetary latitude at which the diapir sinks. We impose a classical Hadamard flow solution for the motion at the interface between a spherical sinking diapir and a viscous mantle on dynamical simulations that account for rotational and inertial effects in order to model the flow within the diapir. The flows are confined to a velocity layer with a thickness that decreases with increasing rotation rate. These $3 \mathrm{D}$ flows are is then used as input for kinematic dynamo simulations to determine the critical magnetic Reynolds number for dynamo onset. Our results demonstrate that the flow pattern inside a diapir sinking into a rotating planet can generate a magnetic field. Large diapirs $(R>10 \mathrm{~km})$ sinking in a mantle with a viscosity ranging from $10^{9}$ to $10^{14} \mathrm{~Pa}$.s provide plausible conditions for a dynamo. Equatorial sinking diapirs are confined to a thicker velocity layer and are thus possibly more favorable for dynamo generation than polar sinking diapirs. In addition equatorial sinking diapirs produce stronger saturated magnetic fields. However, for the range of parameters studied here, estimation of the intensity of diapir-driven magnetic fields suggests that they could not have contributed to the lunar or Martian crustal paleomagnetic fields.

Citation: Monteux, J., N. Schaeffer, H. Amit, and P. Cardin (2012), Can a sinking metallic diapir generate a dynamo?, J. Geophys. Res., 117, E10005, doi:10.1029/2012JE004075.

\section{Introduction}

[2] Episodic migration of large volumes of iron may have strongly contributed to core formations during early planetary evolution [Stevenson, 1981]. Complete separation of the metallic phase from the silicate chondritic material may occur within only several million years on terrestrial planets [Kleine et al., 2002; Yin et al., 2002; Touboul et al., 2007]. Such a fast process necessarily involves melting of the metallic phase which requires a large amount of energy. This energy can be provided by radiogenic heating [Yoshino et al., 2003], impact processes [Tonks and Melosh, 1993] or gravitational energy release during metal-silicate separation [Šrámek et al., 2010]. Once molten, the dense metallic phase can sink toward the center of the planet via various phenomena such as percolation through a solid matrix [Shannon and Agee, 1996], settling of metallic droplets within a convecting magma ocean [Rubie et al., 2003; Höink et al., 2006],

\footnotetext{
${ }^{1}$ Laboratoire de Planétologie et de Géodynamique, UMR 6112, CNRS, Université de Nantes, Nantes Atlantiques Universités, Nantes, France.

${ }^{2}$ ISTerre, Université de Grenoble 1, CNRS, Grenoble, France.

Corresponding author: J. Monteux, Laboratoire de Planétologie et de Géodynamique, UMR 6112, CNRS, Université de Nantes, Nantes Atlantiques Universités, 2 rue de la Houssinière, FR-44000 Nantes, France. (julien.monteux@univ-nantes.fr)

(C2012. American Geophysical Union. All Rights Reserved. 0148-0227/12/2012JE004075
}

two-phase flow dynamics [Ricard et al., 2009; Šrámek et al., 2010] or Rayleigh-Taylor instabilities [Stevenson, 1981; Honda et al., 1993]. These various processes may lead to a wide range of characteristic length scales [Karato and Murthy, 1997]. Percolation and metal rainfall occur at small length scales $\left(\sim 10^{-2} \mathrm{~m}\right)$ and can ultimately form a dense metallic layer at the bottom of a magma ocean. Then, depending on the thickness of this accumulated metallic layer, RayleighTaylor instabilities can mobilize up to $100-\mathrm{km}$ sized diapirs [Honda et al., 1993].

[3] Large scale metallic volumes can also be separated from silicates by differentiation events following a large impact on an undifferentiated planet [Tonks and Melosh, 1993; Monteux et al., 2009]. Indeed, after a large impact a fraction of the kinetic energy is transferred at depth to thermal energy via shock waves propagation and may separate locally the metallic phase from the silicate phase. Then, the dense metallic phase overcomes viscous heating during its sinking toward the center of the planet. If the separated metallic volume is hot enough, vigorous convection occurs within the metallic phase once it has reached the center of the planet, and a dynamo can be generated [Monteux et al., 2011a]. Ultimately, large iron cores of planetesimals could even have directly sunk into the pre-existing core just after an impact [Benz et al., 1987; Monteux et al., 2011b]. In this study, we term metallic diapir every volume of iron that sinks toward the center of the growing planet independently of its size or sinking process. 
[4] Large metallic diapirs lead to a large deviatoric stress that deforms the surrounding material and accommodates the sinking of the diapir toward the center of the planet. Depending on the rheology of the surrounding mantle, negative metallic diapirism may lead to viscous deformation [Monteux et al., 2009], non-linear rheology [Samuel and Tackley, 2008], elasto-plastic deformation [Gerya and Yuen, 2007] or even to fracturing if the ultimate strength of solid rocks is exceeded [Davies, 1982; Stevenson, 2003]. During the early times of planetary formation extinct radioactivities $\left({ }^{26} \mathrm{Al}\right.$ and $\left.{ }^{60} \mathrm{Fe}\right)$ and large impact heating have played a major role in the thermal budget of the planets. This early heating was retained in the internal part of the planet, leading to a hot proto-mantle and favoring sinking via viscous deformation. Hence, large metallic diapirs potentially sank toward the center of the planet in a Hadamard-regime [Hadamard, 1911] with a timescale that is a function of the viscosity of the protomantle [Karato and Murthy, 1997]. This Hadamard flow constrains the internal dynamics of the diapir.

[5] Can a sinking metallic diapir generate a dynamo? Dynamo theory requires three main conditions for the generation of magnetic field: a fluid with large electrical conductivity, large velocities and some favorable flow pattern. In large-scale dynamos operating in planetary outer cores, the energy source generating large velocities is typically thermochemical convection driven by secular cooling and light element release due to a freezing inner core [Olson, 2007]. However, non-convective energy sources may also drive dynamos, for example precession [Tilgner, 2005], tidal distortion of the core-mantle boundary (CMB) [Le Bars et al., 2011] or heterogeneous mechanical boundary conditions [Guervilly and Cardin, 2010].

[6] Kinematic dynamos provide vital information about the efficiency of magnetic field generation for different flow morphologies [Gubbins et al., 2000a, 2000b]. Very simple laminar flows often lack significant helicity which is an important ingredient for a dynamo [Moffatt, 1978]. They are therefore inefficient in generating a magnetic field, which requires the magnetic Reynolds number to exceed large critical values [Gailitis, 1970; Dudley and James, 1989; Moss, 2008]. Even if the helicity is not indispensable in the dynamo process, these kinematic dynamo models have shown that it is a favorable factor. Self-consistent 3D numerical dynamo models show a hemispheric anti-symmetric pattern of helicity associated with the $\alpha^{2}$ dynamo mechanism [Olson et al., 1999]. In these models helicity is generated by the action of the Coriolis force that yields positive/negative radial vorticity correlated with downwelling in the northern/southern hemisphere respectively [Olson et al., 2002; Amit et al., 2007].

[7] We use kinematic dynamo simulations to determine the critical magnetic Reynolds number above which the flow strength (for a given flow pattern) is sufficient to amplify a magnetic seed field. To resolve the flow inside the diapir, we impose the simple flow pattern of a viscous bubble as a mechanical outer boundary condition on a dynamical simulation. Because the diapir is in a rotating planetary reference frame and its low viscosity may accommodate inertial effects, we take into account Coriolis and inertial forces. These rotational and inertial effects may help dynamo action by introducing helicity to the dynamics within the interior of the sinking diapir.
[8] The aim of this study is to determine whether such sinking dynamics can drive a dynamo, and to constrain the required conditions for the size of the diapir and the mantle viscosity of the planet. In addition, we also investigate the importance of the planetary latitude at which the diapir sinks on the conditions for obtaining a dynamo. In section 2 , we show that there exists a considerable range of parameters in which large metallic diapirs are stable. The theory and method are described in section 3 . We present the results of our models in section 4 . In section 5 we discuss the diapir driven magnetic field growth rate and intensity from an initial interplanetary magnetic seed field. In section 6 we discuss the feasibility of obtaining diapir driven dynamos in geophysical conditions. Conclusions and possible planetary implications are highlighted in section 7 .

\section{Stability of Large Metallic Diapirs}

[9] In the laminar flow regime, the sinking velocity of a metallic diapir obeys a Hadamard velocity [Hadamard, 1911; Batchelor, 1967; Monteux et al., 2009]

$$
U=\frac{2}{9}\left(\frac{\eta+\eta_{s}}{\eta+\frac{2}{3} \eta_{s}}\right) \frac{\Delta \rho g R^{2}}{\eta_{s}} \simeq \frac{1}{3} \frac{\Delta \rho g R^{2}}{\eta_{s}}
$$

where $\eta$ is the viscosity inside the diapir, $\eta_{s}$ is the viscosity of the surrounding mantle (with $\eta_{s} \gg \eta$ ), $\Delta \rho$ is the density difference between the metallic diapir and the silicate mantle, $g$ is the gravitational acceleration at the depth of the diapir and $R$ is the radius of the metallic diapir. For early partially molten planetary mantles, $\Delta \rho \sim 5000 \mathrm{~kg} / \mathrm{m}^{3}$ and $\eta_{s} \sim 10^{7}$ $10^{13}$ Pa.s [Karato and Murthy, 1997]. Actually, the gravity and the sinking velocity decrease with depth. For simplicity however, we fix the gravity in the partially molten zone to a constant value of $g \sim 5 \mathrm{~m} / \mathrm{s}^{2}$ (intermediate value between the Moon's and the Earth's surface gravities). The sinking velocity from equation (1) is related to the Stokes velocity by $U_{\text {Stokes }}=2 U / 3$ which is the velocity of a solid sphere sinking in a viscous medium (i.e. $\eta_{s} \ll \eta$ ).

[10] Whether a large metallic diapir will break depends on the stability of the surrounding flow, which is depicted by the Reynolds number $\operatorname{Re}_{s}$ based on the sinking velocity $U$ and the fluid properties of the surrounding mantle:

$$
\operatorname{Re}_{s}=\frac{\rho_{s} U R}{\eta_{s}}=\frac{\rho_{s} \Delta \rho g R^{3}}{3 \eta_{s}^{2}}
$$

where $\rho_{s} \sim 5000 \mathrm{~kg} / \mathrm{m}^{3}$ is the estimated density of the mantle. For $\operatorname{Re}_{s} \gtrsim 10^{3}$, viscous forces are negligible compared to inertia and diapir break-up occurs. When $1 \lesssim R e_{s} \lesssim 10^{3}$, the diapir may or may not break depending on the importance of inertia over surface tension forces. For $R e_{s} \lesssim 1$, inertia is negligible and no break-up occurs [Samuel, 2012]. In Figure 1, we represent the stability domain for large metallic diapirs as a function of diapir size $R$ and mantle viscosity $\eta_{s}$. The conservative upper bound $R e_{s}=10$ plotted in Figure 1 provides a limit on candidate diapirs for dynamo action. Figure 1 shows that even very large diapirs can sink without break-up if the molten mantle viscosity is sufficiently large.

[11] Because the mantle viscosity is strongly dependent on the temperature, the thermal evolution of the protoplanet is a 


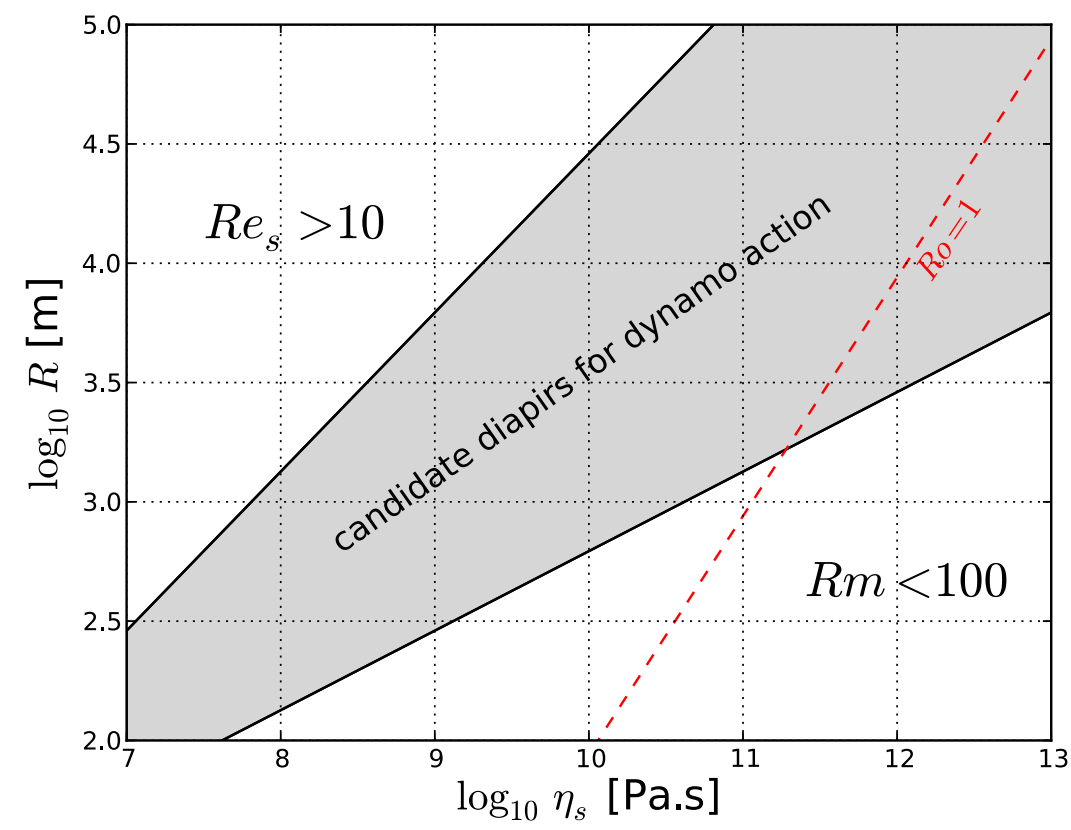

Figure 1. Candidate diapirs for possible dynamo action (shaded region) must have $R e_{s} \lesssim 10$ to be stable and $R m \gtrsim 100$ for magnetic induction to be possible. The $R o=1$ contour shows that larger $\eta_{s}$ gives more dominant rotational effects in the fluid dynamics inside the diapir (above this line $R o>1$ ). For definitions of $R e_{s}, R m$ and $R o$ see equations (2), (3) and (7).

key parameter to define the plausible metal diapir sinking regimes. In the early stages of planetary formation and before the dissipation of potential energy associated with the full core formation, the growing planets were schematically divided in three domains: a completely molten shallow magma ocean, a partially molten deep magma ocean and a solid core [Karato and Murthy, 1997].

[12] The most superficial layer of a growing planet was probably completely molten forming a several hundred kilometers thick magma ocean. In this magma ocean, the viscosity ranges $10^{-4}-10^{2} \mathrm{~Pa}$.s. In the shallow magma ocean and considering $R>1 \mathrm{~km}, R e_{s} \gg 10^{5}$ and turbulent effects lead to emulsification and to rapid break-up of the metallic diapir until it reaches a cm size [Rubie et al., 2003; Samuel, 2012]. However, Dahl and Stevenson [2010] have shown that diapirs with radii larger than $10 \mathrm{~km}$ can survive complete erosion and preserve most of their initial volume as they reach the bottom of a $1000 \mathrm{~km}$ thick magma ocean. In the turbulent magma ocean, the metallic material rapidly segregates from silicates and forms a layer above the rheological transition separating the fully molten magma ocean to the partially molten magma ocean [Höink et al., 2005]. From the iron layer cumulated at the bottom of the molten magma ocean, Rayleigh-Taylor instabilities can mobilize large volume of iron by diapirism [Stevenson, 1981; Honda et al., 1993].

[13] From the bottom of the outermost magma ocean, temperature and melting fraction decrease with depth [Safronov, 1978; Kaula, 1979; Senshu et al., 2002] and the viscosity rapidly increases to $10^{7}-10^{13} \mathrm{~Pa} . \mathrm{s}$ [Karato and Murthy, 1997] (see Table 1). Assuming that $U$ is a Stokes velocity, for a $100 \mathrm{~km}$ radius diapir the typical value of $R e_{s}$ within this partially molten reservoir ranges between $10^{9}$ and $10^{-3}$, while for a $1 \mathrm{~km}$ radius diapir $R e_{s}$ ranges between $10^{3}$ and $10^{-9}$. Hence, in this zone and with a favorable diapir size and viscosity regime, diapirs can potentially settle in a laminar flow regime (see Figure 1). Within the solid deep core, the viscosity was probably larger than $10^{18} \mathrm{~Pa}$.s thus also leading to a laminar Stokes flow regime.

[14] Severe heatings associated with large impacts are phenomena that often occur especially at the end of planetary accretion. In a local volume deep below the impact site, the temperature dramatically increases up to the solidus temperature of silicates which results in massive melting. If the molten volume is not differentiated before the impact, a local separation occurs in a nearly spherical volume [Tonks and Melosh, 1992; Monteux et al., 2009]. The impactor's core material can eventually be collected in the impact-produced magma pool where the local $R e_{s} \gg 1$ [Deguen et al., 2011]. Then, the sinking of this large collected volume is governed by the rheology of the surrounding material and may occur in a laminar Stokes flow regime depending on the viscosity of the mantle. We restrict our study to the laminar flow regime

Table 1. Reference Values for Some Diapirs of Radius $R$ Sinking in a Mantle of Viscosity $\eta_{s}$, Which Are Possible Candidates for Driving a Dynamo

\begin{tabular}{lcccccc}
\hline$R(\mathrm{~km})$ & $\eta_{s}(\mathrm{~Pa} . \mathrm{s})$ & $U(\mathrm{~m} / \mathrm{s})$ & $R e_{s}$ & $R m$ & $E$ & $R o$ \\
\hline 0.1 & $10^{7}$ & 8 & 0.4 & 400 & $10^{-6}$ & $10^{3}$ \\
1 & $10^{7}$ & 800 & 400 & $4 \times 10^{5}$ & $10^{-8}$ & $10^{4}$ \\
1 & $10^{9}$ & 8 & 0.04 & $4 \times 10^{3}$ & $10^{-8}$ & 100 \\
10 & $10^{9}$ & 800 & 40 & $4 \times 10^{6}$ & $10^{-10}$ & $10^{3}$ \\
10 & $10^{11}$ & 8 & $\ll 1$ & $4 \times 10^{4}$ & $10^{-10}$ & 10 \\
10 & $10^{13}$ & 0.08 & $\ll 1$ & 400 & $10^{-10}$ & 0.1 \\
100 & $10^{11}$ & 800 & 4 & $4 \times 10^{7}$ & $10^{-12}$ & 100 \\
100 & $10^{13}$ & 8 & $\ll 1$ & $4 \times 10^{5}$ & $10^{-12}$ & 1 \\
\hline
\end{tabular}




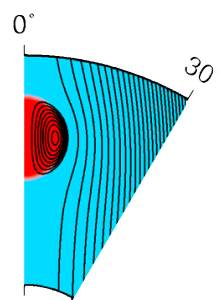

$\eta_{s} / \eta=10^{2}$

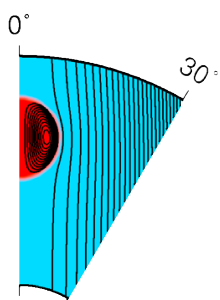

$\eta_{s} / \eta=10^{4}$

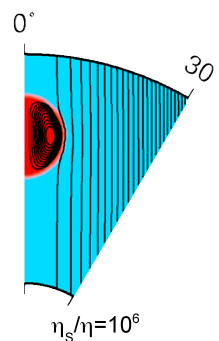

Figure 2. Streamlines of a sinking metallic sphere (red) in an undifferentiated planet (blue). The streamlines are in the moving frame of the sinking diapir. The ratio between the diapir radius to the planetary radius is 0.125 . The three images show the flows for three different ratios between the mantle viscosity $\eta_{s}$ and the diapir viscosity $\eta$. These results were obtained using the thermo-chemical convection code developed by Monteux et al. [2009] in spherical axisymmetric geometry.

where the diapir does not break. Hence, we particularly focus on the dynamics of a diapir sinking in the deep and partially molten magma ocean.

\section{Theory and Method}

[15] The growth or decay of a magnetic seed field $B_{i}$ depends on the diapir magnetic Reynolds number $R m$ which represents the ratio of advection of magnetic field by the flow to magnetic diffusion

$$
R m=\frac{U R}{\lambda}=\frac{\Delta \rho g R^{3}}{3 \eta_{s} \lambda}
$$

with $\lambda \sim 1 \mathrm{~m}^{2} / \mathrm{s}$ being the magnetic diffusivity of liquid iron inside the diapir [Olson, 2007]. Numerical dynamo simulations of rotating convection in a spherical shell show that $R m$ $\gtrsim 100$ is a necessary condition for magnetic field generation [Christensen and Aubert, 2006]. Figure 1 shows the limit $R m=100$ as a function of diapir radius $R$ and mantle viscosity $\eta_{s}$. Combining the conditions on $R e_{s}$ and $R m$, we find a considerable range of diapirs (with $R \gtrsim 100 \mathrm{~m}$ and the full estimated range of $\eta_{s}$ ) that are both stable against break-up as well as sufficiently fast for significant magnetic induction. It is not clear however whether the particular flow pattern inside the diapir is capable of generating a magnetic field. The goal of this study is to verify the feasibility of dynamo action by a diapir sinking flow.

[16] The laminar flow pattern within a sinking diapir in a nonrotating system is well known from theoretical [Hadamard, 1911; Batchelor, 1967] and experimental [Spells, 1952] studies. This circulation pattern consists of a purely poloidal oneroll axisymmetric velocity field [Batchelor, 1967]:

$$
\begin{gathered}
U_{r}(r, \theta)=U\left(R^{2}-r^{2}\right) \cos \theta \\
U_{\theta}(r, \theta)=-U\left(R^{2}-2 r^{2}\right) \sin \theta
\end{gathered}
$$

where $r$ and $\theta$ are the radial and co-latitude spherical coordinates in the reference frame of the diapir. During its sinking, a spherical diapir might deform depending on the rheological properties of both the metallic and the silicate phases [Monteux et al., 2009; Ulvrová et al., 2011]. For simplicity we assume that the diapir maintains a spherical shape during its sinking. Figure 2 shows the internal circulation obtained using the thermo-chemical convection code developed by Monteux et al. [2009]. The flow pattern is in good agreement with both the theoretical and the experimental results and weakly depends on the viscosity ratio between the sinking metallic diapir and the surrounding mantle.

[17] When the planet is rapidly rotating and the viscosity of the diapir is sufficiently small, the Coriolis and inertial forces must be taken into account. Two non-dimensional numbers characterize the dynamical system: the ratio of viscous to Coriolis forces is measured by the Ekman number

$$
E=\frac{\nu}{\Omega R^{2}}
$$

where $\nu=\eta / \rho$ is the kinematic viscosity of the molten iron and $\Omega$ is the rotation rate of the planet. The ratio of inertial to Coriolis forces is characterized by the Rossby number

$$
R o=\frac{U}{\Omega R}
$$

where $U$ is the amplitude of the sinking diapir velocity field (equation (1)). The rotation rate $\Omega$ has probably decreased during the early stages of planetary accretion from a couple of hours [Agnor et al., 1999]. For simplicity, we consider in our study a constant $\Omega$ equal to the current Earth value (see Table 2).

[18] We solve numerically the non-dimensional NavierStokes equation including Coriolis and inertial forces in a spherical container representing the sinking diapir:

$$
\frac{\partial \mathbf{u}}{\partial t}+\mathbf{u} \cdot \nabla \mathbf{u}+2 \mathbf{e}_{\mathbf{z}} \times \mathbf{u}=-\nabla \pi+E \nabla^{2} \mathbf{u}
$$

where $\mathbf{u}$ is the velocity field (in units of $R \Omega$ ), $t$ is time (in units of $\left.\Omega^{-1}\right), \mathbf{e}_{\mathbf{z}}$ is the unit vector in the direction of the planetary rotation axis and $\pi$ the reduced pressure. The Hadamard velocity field (equations (4) and (5)) scaled by Ro is imposed at the spherical outer boundary and drives the flow within the diapir. When the hydrodynamic diapir Reynolds number $R e=R o / E \ll 10^{3}$ (not to be confused with $R e_{s}$ ),

Table 2. Estimated Parameters and Non-dimensional Numbers for Present Earth's Outer Core, a Candidate Sinking Diapir, and Input Non-dimensional Numbers Used in Our Models ${ }^{\mathrm{a}}$

\begin{tabular}{lccc}
\hline & Earth & Diapir & Models \\
\hline$\nu$ & $10^{-6}$ & $10^{-6}$ & - \\
$\lambda$ & 1 & 1 & - \\
$R$ & $3 \times 10^{6}$ & $10^{2}-10^{5}$ & - \\
$\Omega$ & $7 \times 10^{-5}$ & $7 \times 10^{-5}$ & - \\
$U$ & $5 \times 10^{-4}$ & $10^{-5}-10^{3}$ & - \\
$E$ & $10^{-15}$ & $10^{-12}-10^{-6}$ & $10^{-5}-10^{-2}$ \\
$R o$ & $10^{-6}$ & $10^{-3}-10^{4}$ & $10^{-3}-1$ \\
$R m$ & $10^{3}$ & $10^{2}-10^{8}$ & $10^{3}-10^{5}$ \\
$P m$ & $10^{-6}$ & $10^{-6}$ & $10^{-1}-10^{5}$ \\
\hline
\end{tabular}

${ }^{\text {a}}$ All parameters are given in SI units. All values for Earth's core are from Olson [2007]. Diffusivities and rotation rate for diapirs are assumed to be as for the Earth's core. Note that in calculating the non-dimensional number ranges for the diapir not all combinations of $R$ and $U$ are admissible, since $U$ depends on $R$ (equation (1)). For the candidate sinking diapir, see Figure 1. 


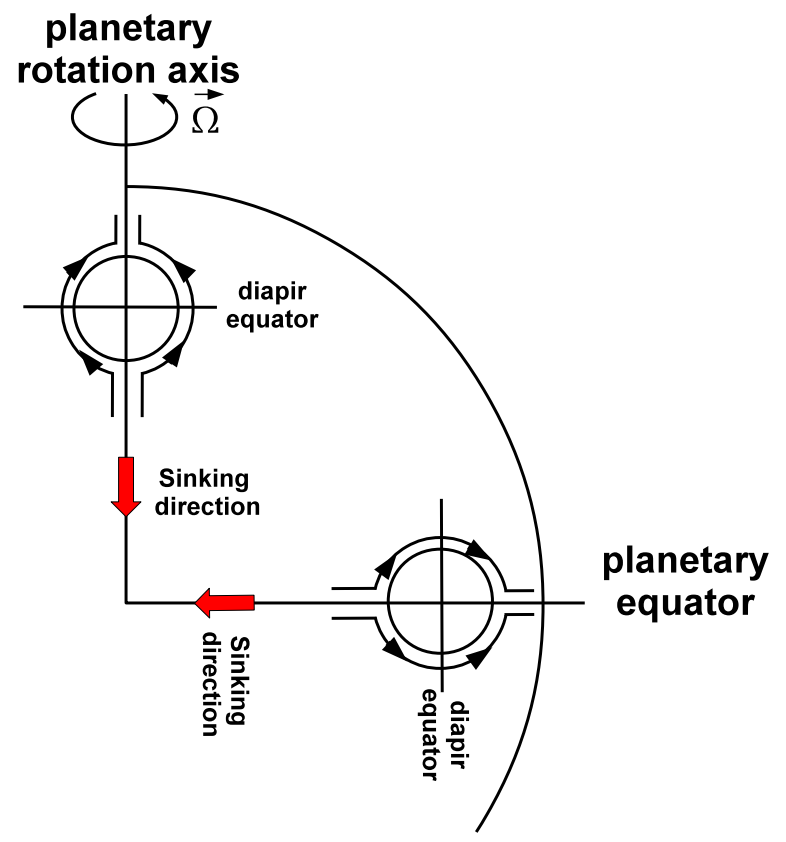

Figure 3. Schematic illustration of the geometries of the two end-member sinking diapirs. In polar sinking (top) the local gravity is parallel to the rotation vector, whereas in equatorial sinking (right) the local gravity is perpendicular to the rotation vector.

the expected flow pattern equations (4) and (5) is recovered in the whole volume to a very good accuracy. Details of the numerical method can be found in Appendix A.

[19] If the resulting three-dimensional flow $\mathbf{u}$ is stationary, it is then used as input to a kinematic dynamo code, which solves the induction equation

$$
\frac{\partial \mathbf{B}}{\partial t}=\nabla \times(\mathbf{u} \times \mathbf{B})+\frac{E}{P m} \nabla^{2} \mathbf{B}
$$

where the magnetic field $\mathbf{B}$ is in units of $\sqrt{\mu_{0} \rho_{F e}} R \Omega$, and $P m=\nu / \lambda$ is the magnetic Prandtl number. $\mu_{0}$ is the magnetic permeability of free space $\left(\mu_{0}=4 \pi \times 10^{-7} \mathrm{H}^{-m^{-1}}\right)$ and $\rho_{F e}$ is the diapir density $\left(\rho_{F e}=10^{4} \mathrm{~kg} \cdot \mathrm{m}^{-3}\right)$. Note that $R m$ (equation (3)) can be rewritten as $R m=R o P m / E$. A growing magnetic field indicates the presence of a dynamo.

[20] If the Navier-Stokes simulation leads to a timedependent flow (as it is the case for high $R o$ and $R e$ ), we resort to a much slower fully self-consistent dynamo code, which solves the induction equation (9) coupled with the Navier-Stokes equation (8) with the addition of the Lorentz force $(\nabla \times \mathbf{B}) \times \mathbf{B}$. We denote the critical magnetic Reynolds number for the onset of a dynamo by $R m_{c}$. To characterize the efficiency of the flow pattern in generating a magnetic field, we also examine the helicity $H=\mathbf{u} \cdot(\nabla \times \mathbf{u})$.

[21] The relevant parameters and non-dimensional numbers are listed in Table 2, including their typical values for Earth's outer core, the possible ranges for the sinking diapir, as well as values used in our numerical models. The most significant variability and uncertainty appears in the size of the diapir $R$ and the viscosity of the surrounding mantle $\eta_{s}$. It is therefore worth-while writing the control parameters of the models in terms of these two quantities. For example, substituting equation (1) into equation (7) allows rewriting the Rossby number as

$$
R o=\frac{1}{3} \frac{\Delta \rho g R}{\eta_{s} \Omega}
$$

Using fixed values for $\Delta \rho, g, \Omega, \nu$ and $\lambda$, the input control parameters $E, R o$ and $R m$ are given in terms of the diapir size $R$ and the mantle viscosity $\eta_{s}$ in Tables 1 and 2. For a successful dynamo $\left(R m>R m_{c}\right)$ to be considered geophysical, it is required to have $E, R o$ and $R m$ within the (rather broad) estimated range for candidate diapirs (see Figure 1), but also the magnetic Prandtl number must have the value of molten iron, that is $P m \sim 10^{-6}$ [Olson, 2007]. We limit our dynamical models to the rotational regime of $R o \leq 1$ but we also investigate the ability of the simple laminar Hadamard flow without rotation and inertia to generate a magnetic field.

[22] Two end-member scenarios are examined, one in which the diapir falls on the geographic pole of the planet, the other in which the diapir falls on the equator of the planet. In the first the Hadamard flow forcing is parallel to the planetary rotation axis, in the second the Hadamard flow forcing is perpendicular to the planetary rotation axis (see Figure 3). The consequences of these two end-member scenarios for the dynamo are compared.

\section{Results}

\subsection{Fluid Flow Inside the Diapir}

[23] We begin by reporting the velocity fields within the diapir obtained by our dynamical model. Figure 4 a shows a solution for a large Ekman number of $E=10^{-2}$ and a Rossby number of $R o=10^{-1}$ for a polar sinking diapir. The meridional flow consists of a single poloidal roll anti-symmetric with respect to the equator and axisymmetric with respect to the rotation axis, as in the classical Hadamard flow solution without rotation and inertia (equations (4) and (5)). However, in addition the solution contains an azimuthal axisymmetric toroidal flow component anti-symmetric with respect to the equator, which is due to the Coriolis force. Compared to Figure 2, the streamlines are distorted toward the outer part of the sphere in response to the rotational effect. Note that the radial flow is much weaker than the tangential flow. In the rotational regime where $R o \ll 1$ the flow pattern is weakly dependent on $R o$. In this regime, the main difference between flow models of two different $R o$ values is that the magnitude scales linearly with $R o$. For $R o \sim 1$ and hence large Reynolds numbers $R e=R o / E$, the flow is no longer stationary.

[24] For smaller Ekman numbers (Figures $4 \mathrm{~b}$ and 4c), the flow pattern is qualitatively similar but is now confined to a thinner layer below the boundary of the diapir. Figure 5 shows the thickness of the velocity layer $h$ normalized by the diapir's radius as a function of $E^{-1}$ for $R o=10^{-2}$. The decrease in $h$ with decreasing $E$ obeys a power law

$$
\frac{h}{R}=A E^{x}
$$

For a polar sinking diapir the prefactor is $A_{p} \sim 4$ and the power is $x_{p} \sim 0.5$ (Figure 5 , red). The $1 / 2$ power is suggestive of an Ekman boundary layer control [e.g. Pedlosky, 1987], as may be expected in a system governed by rotational effects. The same results are found for all $R o<1$ values (not shown). 


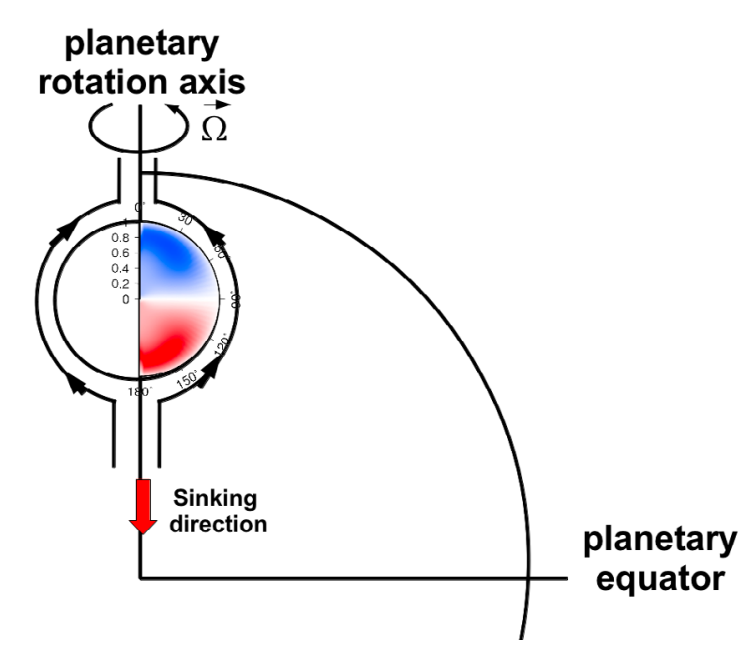

a) $\begin{aligned} & \mathrm{Ro}=10^{-1} \\ & \mathrm{E}=10^{-2}\end{aligned}$

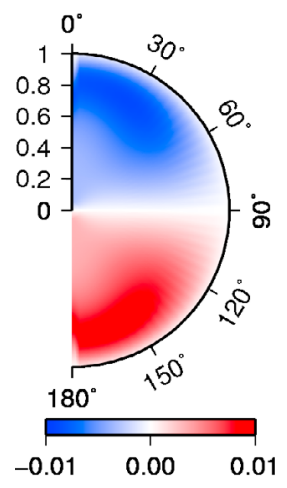

b) $\mathrm{Ro}=10^{-1}$ $E=10^{-3}$ c) $\mathrm{Ro}=10^{-1}$ $E=10^{-4}$
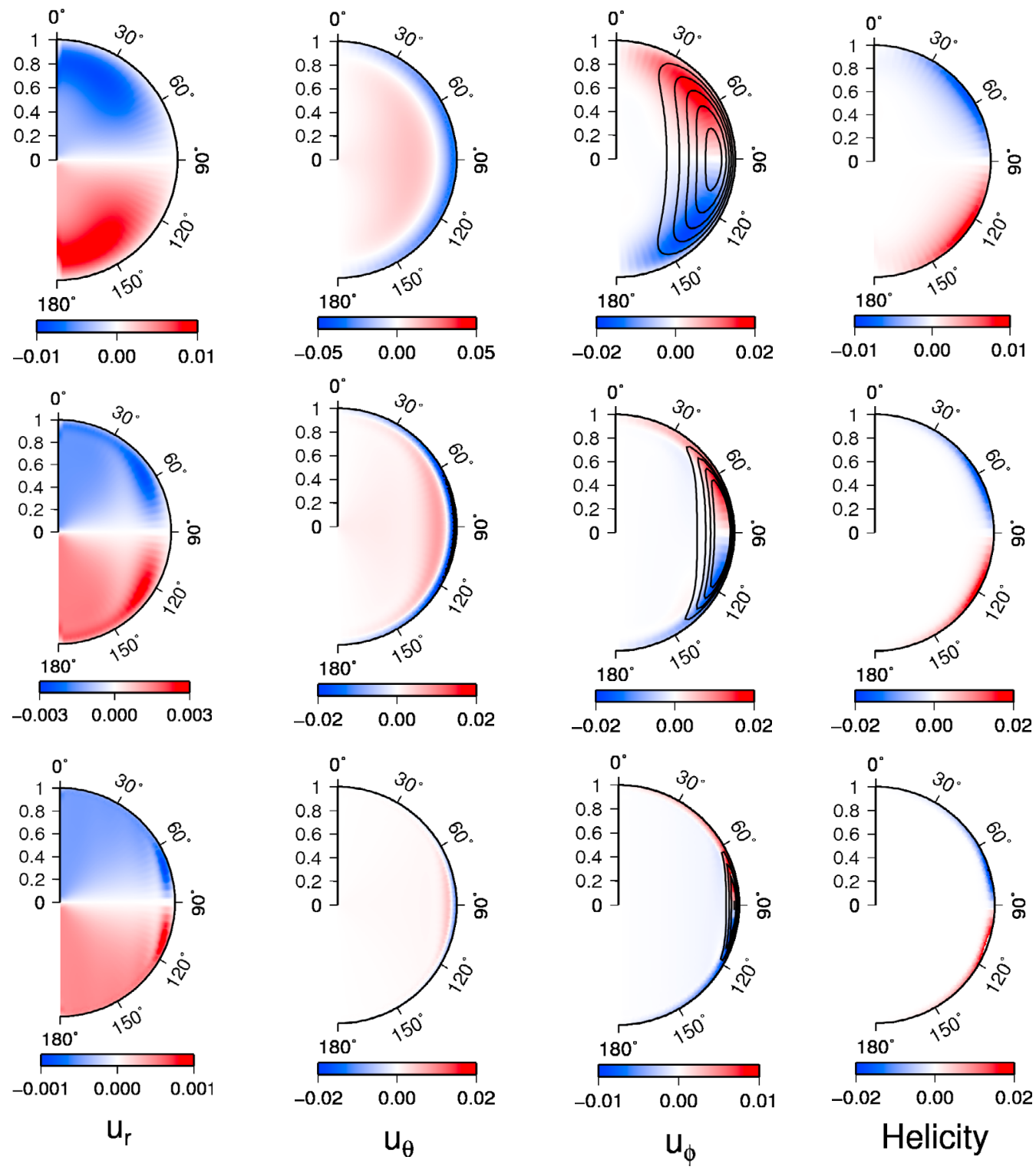

Figure 4. Flow components and helicity for three different $E$ values and $R o=10^{-1}$ in the case of a polar sinking diapir. The streamlines with black solid contours (representing counter-clockwise circulation) are superimposed on the $u_{\phi}$ subplots. The sinking geometry is illustrated at the top of the figure where $u_{r}$ is represented. The mean flow is axisymmetric. 


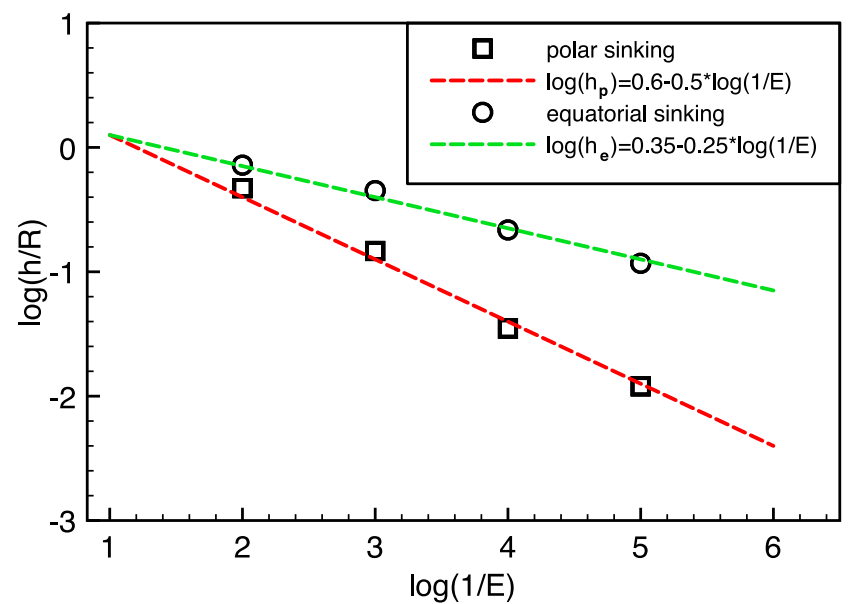

Figure 5. The non-dimensional thickness of the velocity layer $h / R$ as a function of $E^{-1}$ for $R o=10^{-2}$ in log-log scale. The depth is defined where the azimuthal velocity drops to $10 \%$ of its maximum value at colatitude $60^{\circ}$ (for polar sinking diapir, red) or at co-latitude $90^{\circ}$ (for equatorial sinking diapir, green).

[25] We repeat the flow analysis for equatorial sinking diapirs. Note that the visualization is in the planetary reference frame, so the north pole points in the direction of the rotation rate (and not in the diapir sinking latitude). Here the flow is symmetric with respect to the equator (Figure 6). In this configuration, the rotation and sinking axes are perpendicular, so that the axisymmetry of the flow is lost. Indeed, in the equatorial plane, the stationary flow is dominated by an $m=1$ spiral (see Figure 7), and the effect of the Coriolis force tends to make the flow invariant along the rotation axis (Figure 6). The analysis of the thickness of the velocity layer for equatorial sinking diapir leads to the prefactor $A_{e} \sim 2$ and a power of $x_{e} \sim 0.25$ (equation (11) and Figure 5, green), reminiscent of a Stewartson internal layer control [Stewartson, 1957; Schaeffer and Cardin, 2005].

[26] Because helicity favors dynamo action, we examine the pattern of helicity and its dependence on $E$ and Ro. For a polar sinking diapir, the helicity is hemispheric anti-symmetric negative/positive in the northern/southern hemisphere respectively (Figure 4). This helicity pattern is governed by the interaction between the latitudinal flow and the radial shear of the azimuthal flow. As with the flow pattern, the thickness of the layer of significant helicity decreases with decreasing $E$. For equatorial sinking diapirs the helicity is also hemispheric anti-symmetric (Figure 6). Here the hemispheric antisymmetric helicity pattern is obtained by the interaction between the azimuthal flow and the radial shear of the latitudinal flow.

\subsection{Magnetic Field Generation}

[27] First, we used the analytic Hadamard flow solution without rotation and inertia (equations (4) and (5)). We found no dynamo. Next, we accounted for the effect of rotation: for each flow solution obtained by different combinations of $E$ and $R o$, we search for the critical magnetic Reynolds number $R m_{c}$ above which dynamo action takes place. Figure 8 shows the results of such a parametric study for a polar sinking diapir. The smallest $R m_{c}$ values (most favorable flow morphologies for dynamo action) appear for $E=10^{-3}$. Weaker rotation yields weaker helicity and subsequent field generation, whereas stronger rotation confines the flow to a thinner layer and thus dynamo action is once again less efficient. In the rotational regime where $R o \ll 1, R m_{c}$ does not depend on $R o$. This is expected because the flow pattern is almost independent of $R o$ for $R o \ll 1$. On approach to $R o \sim 1$, the flow pattern changes and some non-trivial dependence of $R m_{c}$ on $R o$ emerges.

[28] The results of the parametric study for the equatorial sinking diapirs (Figure 9) are similar to those for the polar sinking diapirs. Because the flow of equatorial sinking diapirs is not axisymmetric, one must compute the velocity and magnetic fields with many coupled azimuthal modes, leading to much higher computation times (10 to 30 times longer) than in the polar sinking dynamos. Hence, we decided to focus on $E \leq 10^{-3}$ and $0.1 \leq R o<1$. The smallest $R m_{c}$ for equatorial sinking diapir was found for $E=10^{-4}$. From Figures 8 and 9 we can see that $R m$ has to be at least larger than 3000 to envision a diapir driven dynamo generation which restricts the candidate diapir domain (Figures 1 and 10).

[29] Figure 11 shows images of the magnetic field for a diapir sinking from the equator for two sets of parameters $\left(\left(E=10^{-5}, R o=0.3\right)\right.$, top; $\left(E=10^{-4}, R o=0.1\right)$, bottom $)$. The magnetic field is characterized by small scale features as the flow within the diapir becomes more turbulent (Figure 11, bottom). Note that in the equatorial sinking case, even at small Ekman number, the magnetic field is strong in a significant volume fraction, despite the confinement of the flow to a narrower layer.

\section{Magnetic Seed Field Growth Rate and Saturation}

[30] We have demonstrated that a sinking diapir can generate a dynamo. Next we estimate the intensity of the magnetic field, in particular whether the sinking time is long enough for the magnetic field to reach saturation before arriving at the bottom of the partially molten mantle. This requires the computation of the non-linear dynamo problem, i.e. the simultaneous solution of the coupled momentum and induction equation. As initial condition, we consider a uniform interplanetary magnetic seed field $B_{i}=3 \times 10^{-9} \mathrm{~T}$ [Riedler et al., 1989]. We monitor the intensity of the dipolar component of the magnetic field at the diapir surface as a function of time from a successful dynamo model of equatorial sinking diapir. We have computed several such dynamos, all of them exhibiting a similar behavior: strong small scale magnetic field within the diapir (Figure 11), with relatively weak dipolar field at its surface. In Figure 12, the time is non-dimensionalized by the time a diapir with radius $R$ needs to travel a distance $R$. The magnetic field is saturated before a $10 \mathrm{~km}$ diapir reaches the bottom of a $200 \mathrm{~km}$ depth partially molten mantle. For polar diapirs (not shown), the saturated field is slightly weaker and the growth rate is slower compared with the equatorial case.

[31] We also monitor the corresponding magnetic field intensity at the surface of the planet as a function of time (Figure 12, red line). The intensity of the magnetic field 


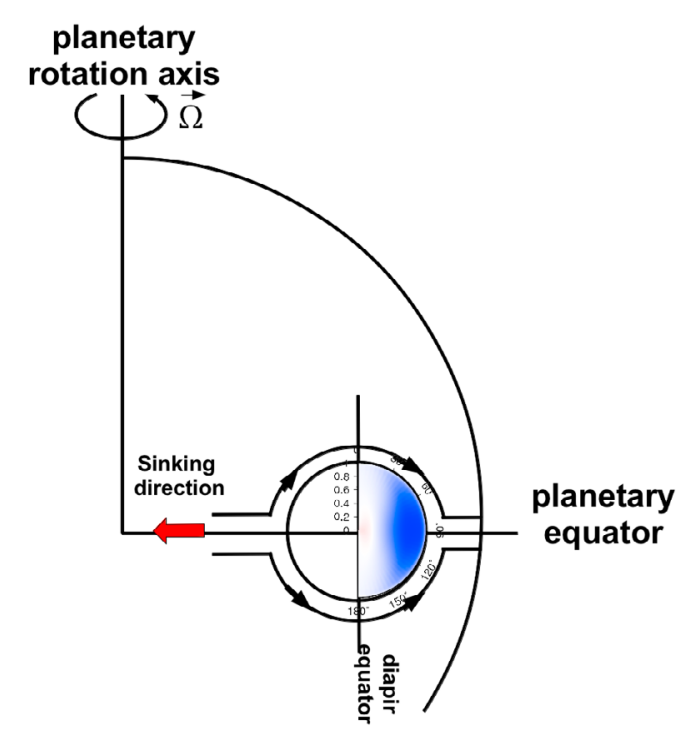

a) $\mathrm{Ro}=10^{-1}$
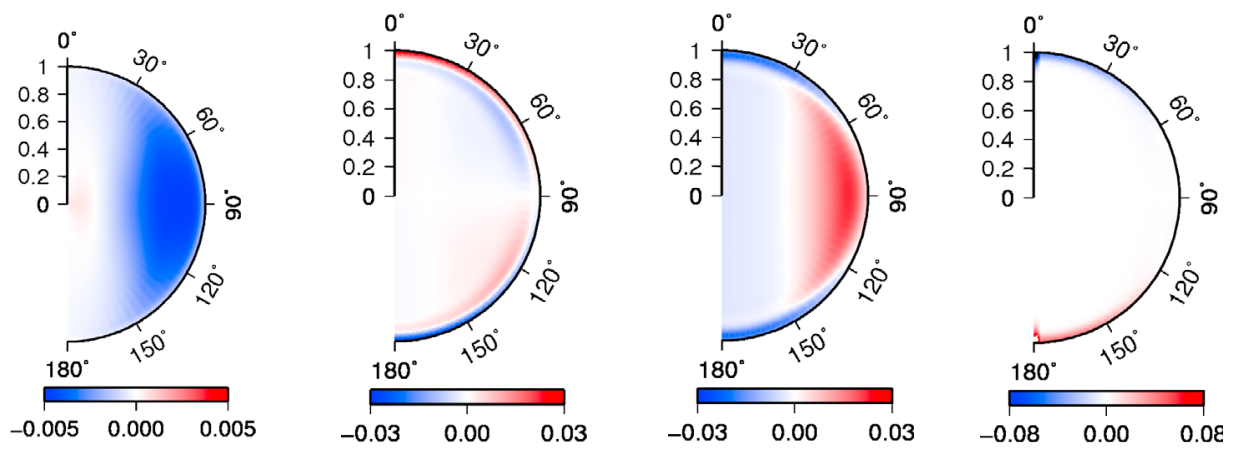

b) $\mathrm{Ro}=10^{-1}$
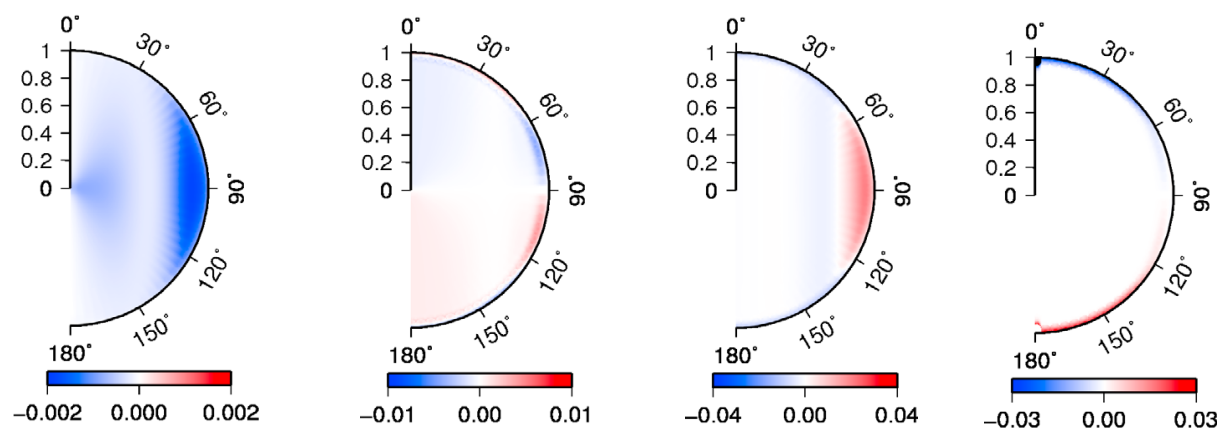

c) $\begin{aligned} & \mathrm{Ro}=10^{-3} \\ & \mathrm{E}=10^{-3}\end{aligned}$
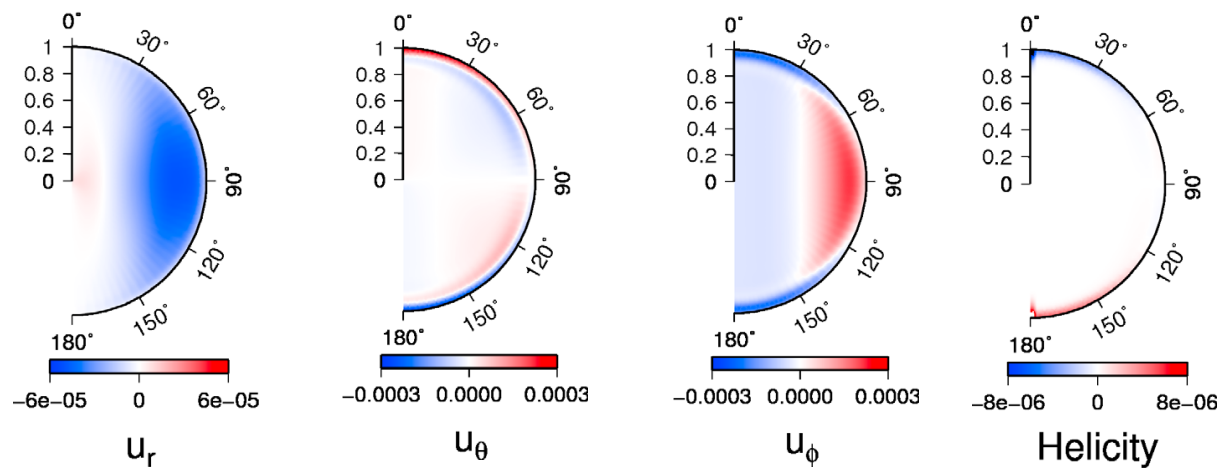

Figure 6. As in Figure 4 for equatorial sinking diapirs. As in Figure 4, the vertical direction points to the rotation axis of the planet. 

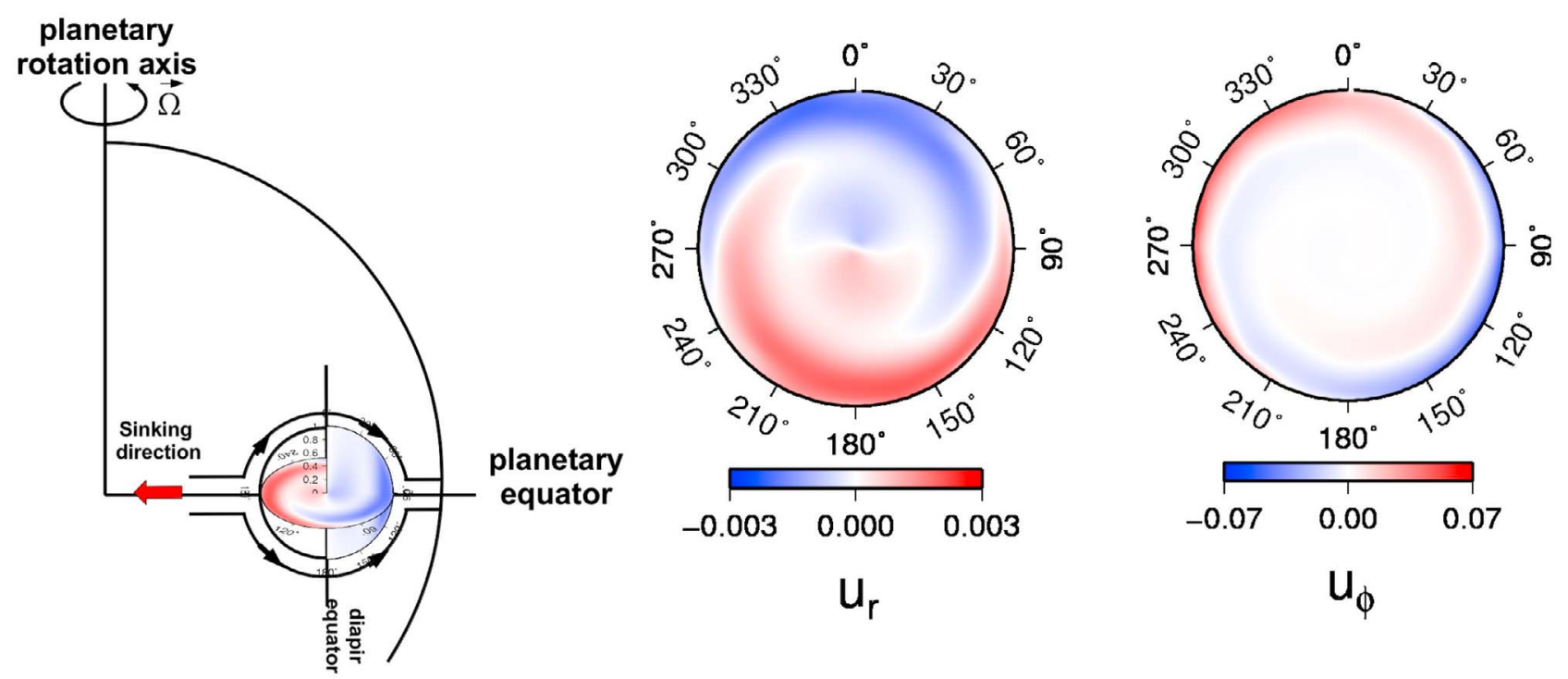

Figure 7. Illustration of the geometry and flow components in the equatorial plane of the planet for $E=10^{-4}$ and $R o=10^{-1}$ for an equatorial sinking diapir.
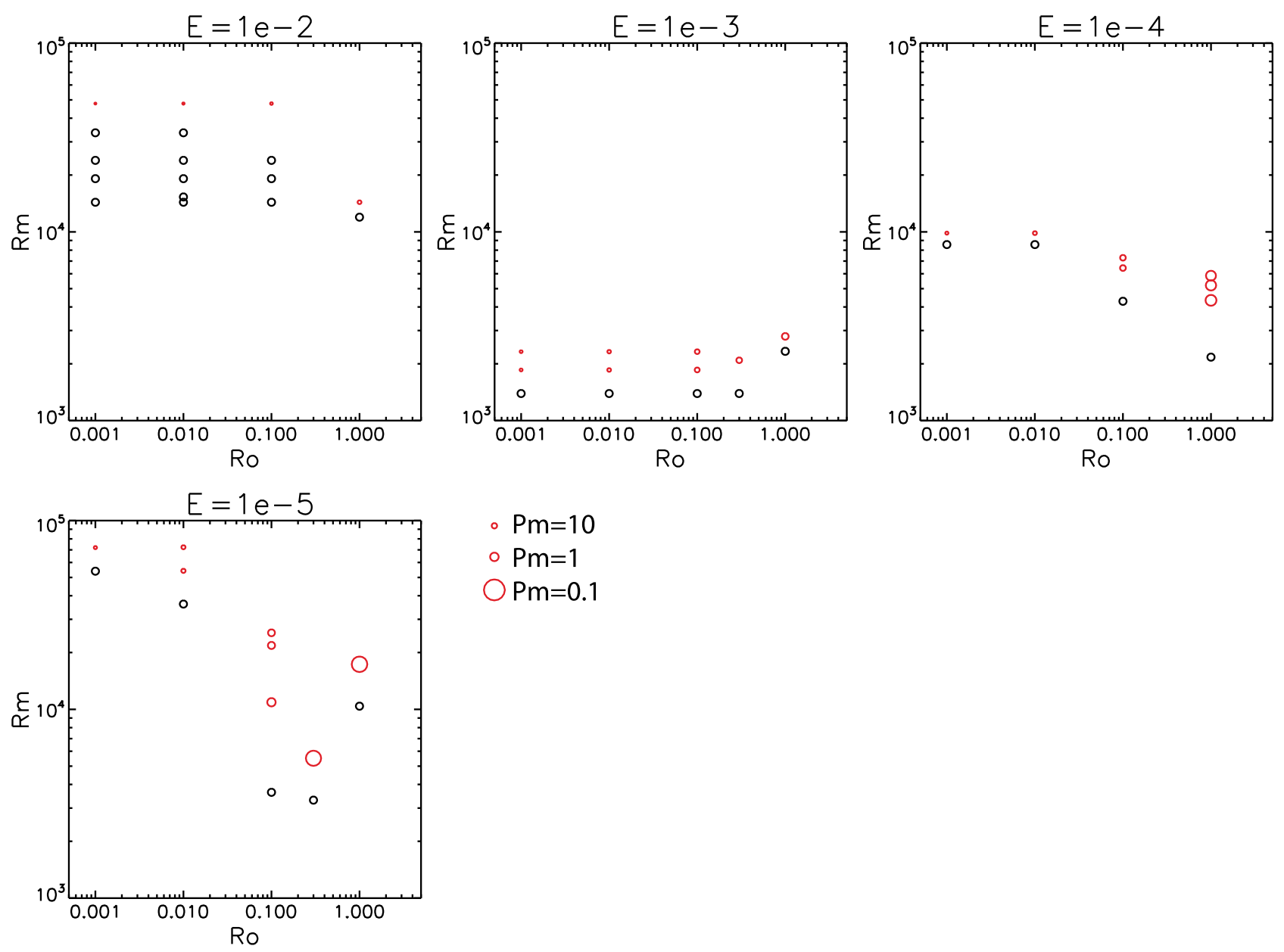

- $\mathrm{Pm}=10$

- $\mathrm{Pm}=1$

$\bigcirc \mathrm{Pm}=0.1$

Figure 8. Regime diagram of kinematic dynamos for polar sinking diapirs. Black circles are no dynamos, red circles are dynamos. The values of $P m$ for the dynamo cases in log-scale are represented by the size of the red circles (see legend). 

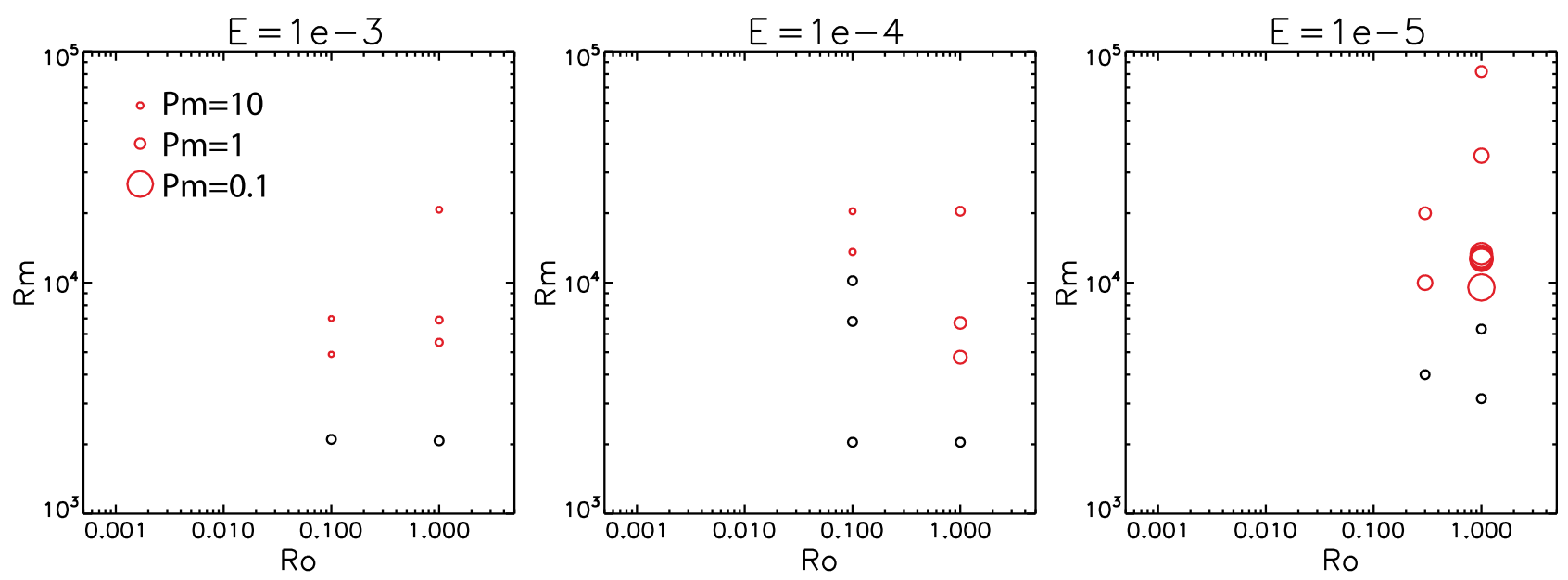

Figure 9. As in Figure 8 for equatorial sinking diapirs.

decreases with the distance $d$ from the surface of the diapir:

$$
B \sim B_{0} \frac{d^{-\ell-2}}{R}
$$

where $\ell$ is the spherical harmonic degree. We consider the dipolar component of the magnetic field (i.e. $\ell=1$ ) which is the strongest contribution far from the generating region. The magnetic field intensity generated by the diapir deep in the mantle never overcomes the initial magnetic seed field intensity. This means that within the range of parameters of our study $\left(R o \leq 1, E k \geq 10^{-5}\right.$ and
$P m \geq 10^{-1}$ ), a dynamo generated by a sinking metallic diapir is unlikely to leave a footprint on the surface paleomagnetic field of a growing planet.

\section{Discussion}

[32] We studied dynamos generated by 3D circulation driven by Hadamard flow on the outer boundary under the influence of the Coriolis force $(R o \lesssim 1, E \ll 1)$. This is an important constraint, which restricts the candidate diapirs from our models to the region below the $R o \simeq 3$ line of Figure 10, where rotational effects are important. In the highly rotational regime of $R o \ll 1$ the flow morphology as

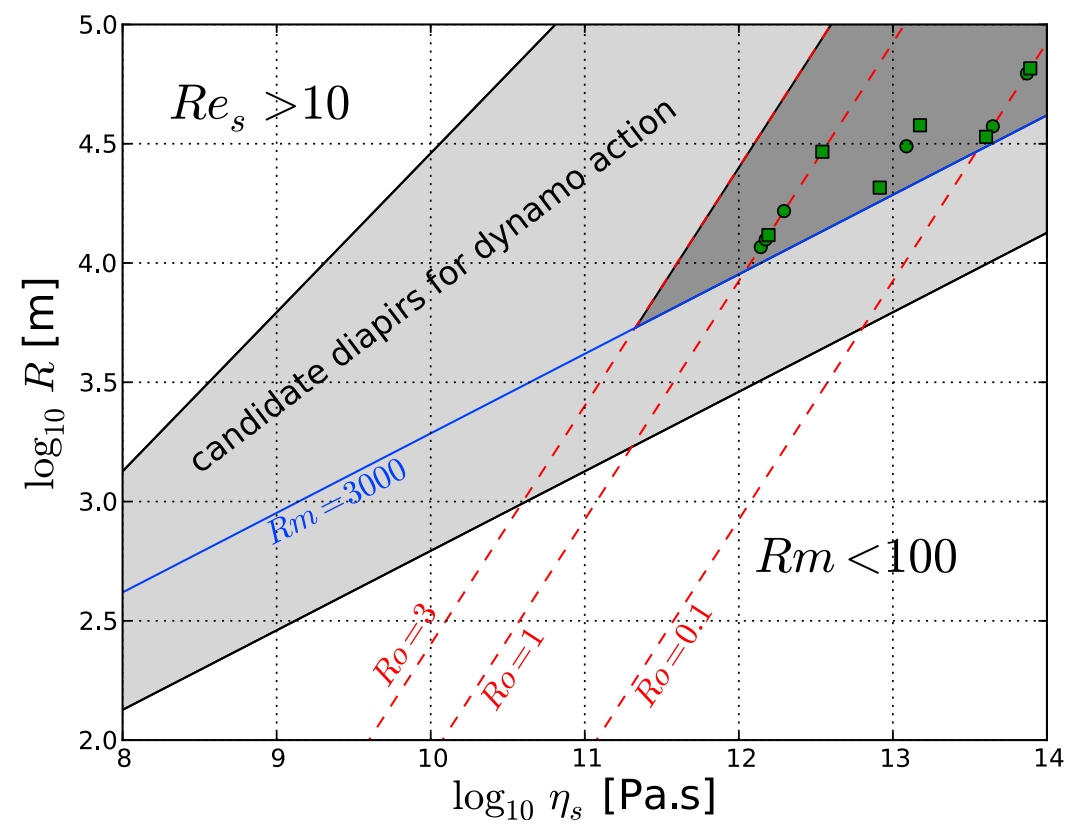

Figure 10. A refined diagram of candidate diapirs for dynamo action. We constrain the candidate diapirs from the results of our models to the dark shaded zone. Successful dynamos are represented with green symbols (squares for polar and circles for equatorial sinking diapirs). The blue solid line for $R m=3000$ represents the minimum value for a successful dynamo from our models. The red dashed line for $R o=1$ represents the limit of the rotational regime investigated in our study. We also represent the $R o=0.1$ value. Larger but moderate $R o$ values marked by the $R o=3$ line (not studied here) may possibly lead to dynamo action. We extend the domain to $\eta_{s}=10^{14} \mathrm{~Pa}$.s where dynamos were observed in our models. 

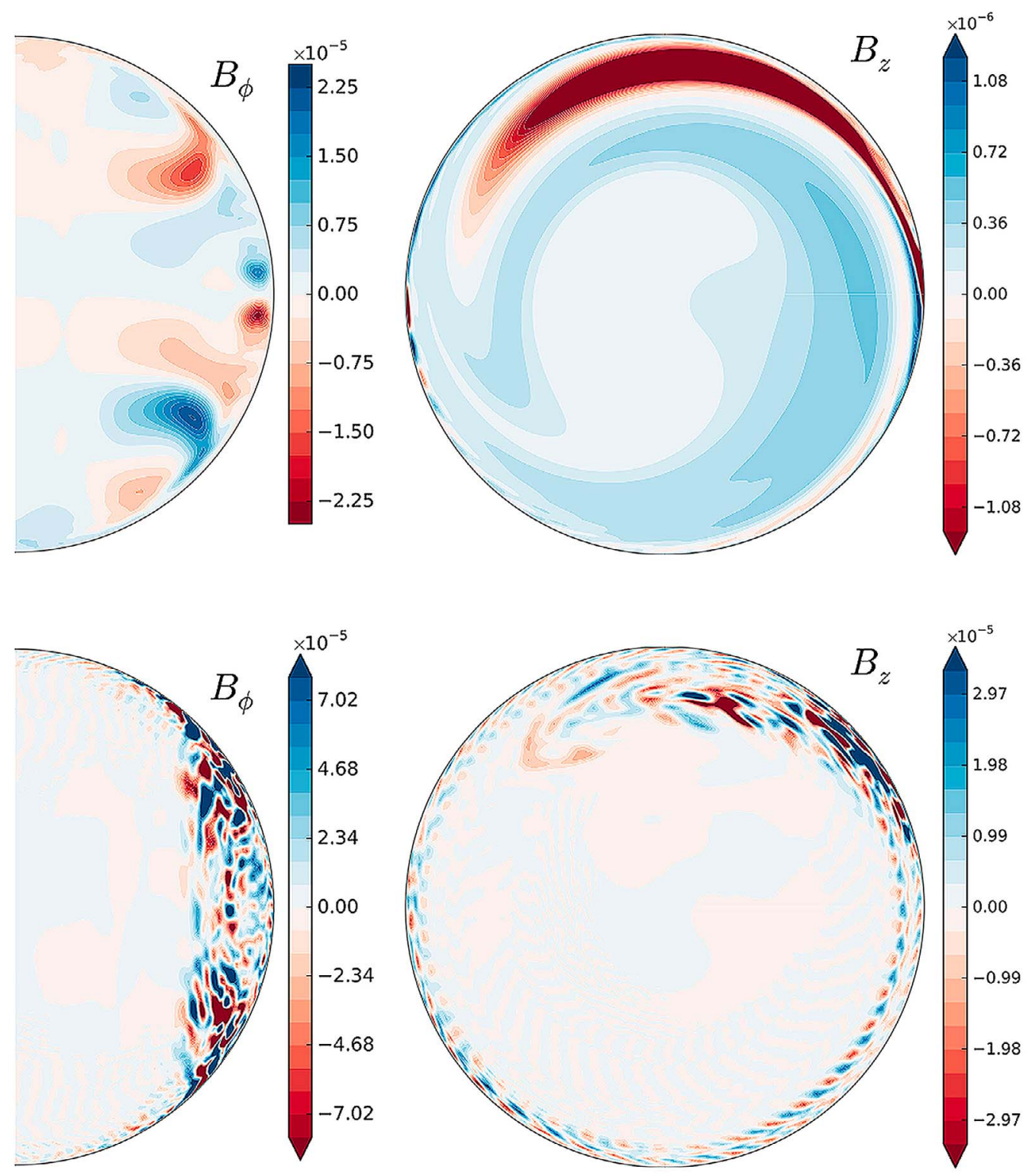

Figure 11. Example of dynamo magnetic fields for an equatorial sinking diapir. (left) The $\phi$-component of the magnetic field in an arbitrary meridional cross-section and (right) the $Z$-component of the magnetic field in the equatorial plane. (top) $E=10^{-4}, R o=0.1, P m=20, R e=10^{3}$ and (bottom) $E=10^{-5}, R o=0.3$, $P m=0.5, \operatorname{Re}=3 \times 10^{4}$.

well as $R m_{c}$ do not depend on $R o$. The behavior of the system for $R o \sim 1$ is complicated to investigate due to computational limitations. In these systems turbulence becomes important, the flow is unsteady and temporal fluctuations dominate the dynamics. This regime can eventually lead to more complex flows and favor dynamo generation, although it is unlikely [Peyrot et al., 2007; Ponty and Plunian, 2011].

[33] Can sinking diapirs produce dynamos in nature? In our numerical simulations, all physical parameters are in the geophysical range except for the liquid iron viscosity, which influences both Ekman and magnetic Prandtl number. Unfortunately, simulations with lower $E$ are computationally very costly. In Figures 8 and 9 the magnetic Prandtl number $P m$ is represented by the size of the circles in the dynamo cases, so that larger circles represent smaller $P m$, closer to the geophysical value. Both these figures show that we have obtained dynamos at smaller $P m$ values when decreasing $E$. In the regime which is accessible for our simulations, the lowest $P m$ values are on the order of 0.1 , five orders of magnitude too large. Such computational problems of obtaining Earth-like $E$ and $P m$ values are ubiquitous to all numerical dynamo studies [Christensen and Aubert, 2006; Christensen and Wicht, 2007].

[34] From Figures 8 and 9, we obtain the critical magnetic Reynolds number $R m_{c}$ for each combination of $E$ and $R o$ values. This global $R m_{c}$ scales with $R^{3}$ (equations (1) and (3)). Lowering the Ekman number confines the flow to a narrow velocity layer at the outer part of the diapir, which results in larger $R m_{c}$. This motivates defining a local critical 


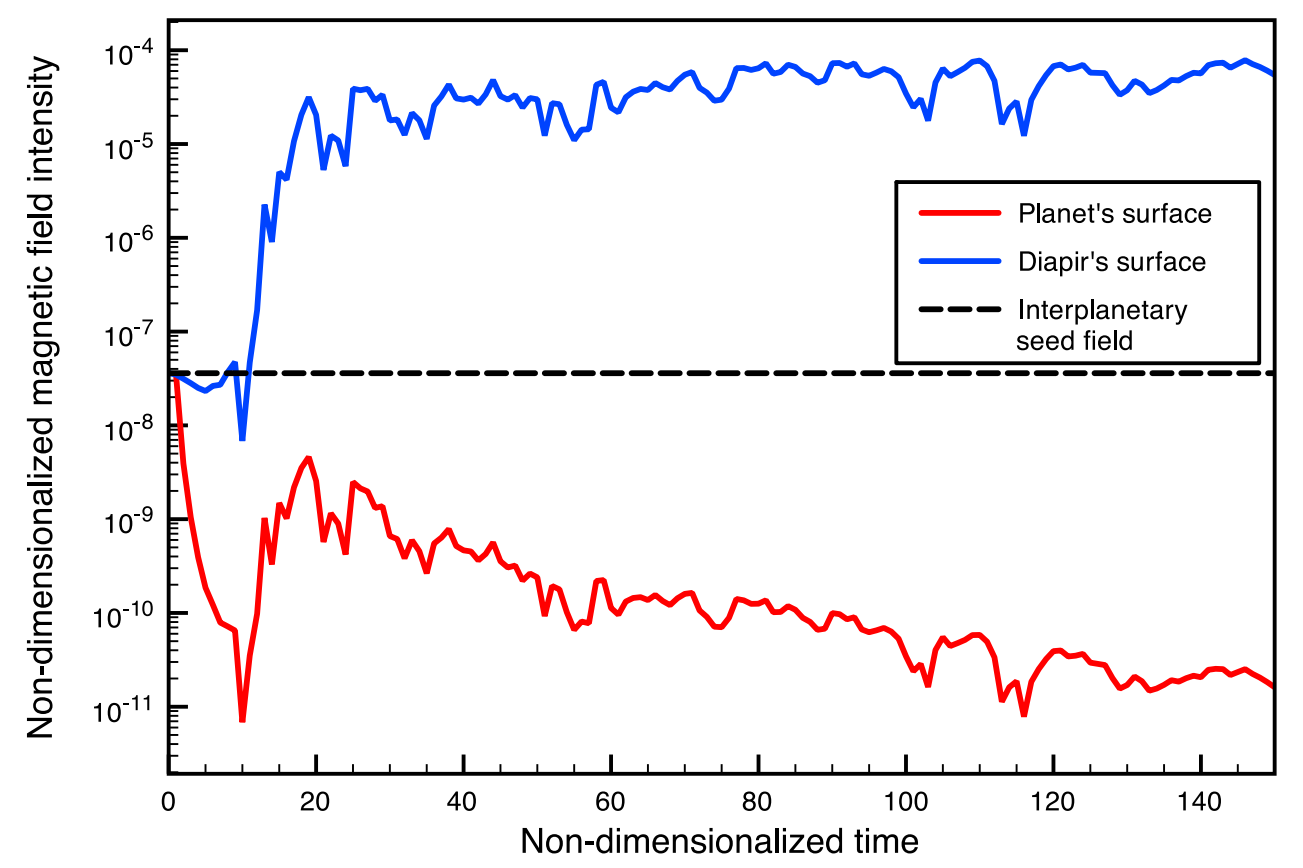

Figure 12. Intensity of the dipolar magnetic field generated by an equatorial sinking diapir as a function of time with $R o=1, E=10^{-5}, R m=10^{5}, P m=1$. The intensity of the magnetic field is non-dimensionalized by $\sqrt{\mu_{0} \rho_{F e}} R \Omega \rho_{F e}=10^{4} \mathrm{~kg} \cdot \mathrm{m}^{-3}$ and $R=10 \mathrm{~km}$. The blue line represents the evolution of the dipolar magnetic field at the surface of the diapir, and the red line at the surface of the planet.

magnetic Reynolds number $R m_{c}^{L}$ based on the thickness of the velocity layer $h$ :

$$
R m_{c}^{L}=\frac{h}{R} R m_{c}
$$

[35] Using the best fits to $h$ from Figure 5, we plot $R m_{c}^{L}$ as a function of $E^{-1}$ in Figure 13. It is difficult to extrapolate a relation between $R m_{c}^{L}$ and $E$. Based on this figure, $R m_{c}^{L}$ appears to have an asymptotic behavior with decreasing $E$, with polar sinking dynamos seeming to be favorable. However, the thickness $h$ of that layer is much larger in the equatorial sinking case $\left(h \sim E^{1 / 4}\right)$ than in the polar sinking case $\left(h \sim E^{1 / 2}\right)$. It thus appears that dynamo generation from a sinking diapir could be favored by equatorial sinking diapirs for realistic Ekman numbers. Even in the very pessimistic case, where $R m_{c} \sim 10^{5}$ (two orders of magnitude larger than our optimal model) for realistic Ekman numbers, giant diapirs (radius from 30 to $100 \mathrm{~km}$ ) can still produce strong magnetic fields during their sinking. In addition, the turbulent fluctuations may limit the decrease of $h$ with decreasing $E$ (Figure 5), leading to the decrease of $R m_{c}$ at low $E$ (equation (13)). However, lowering the Ekman number will lead to smaller scale magnetic field generation (as suggested by Figure 11), leading to a possibly lower magnetic field at the surface of the planet.

\section{Conclusions and Perspectives}

[36] Our results show that the flow pattern driven by a diapir sinking through a partially molten mantle within a rotating planet can generate a magnetic field. This dynamo generation seems more favorable for a diapir sinking from the equator than from the planet's rotational pole. Large diapirs $(R>10 \mathrm{~km})$ sinking in a mantle with a viscosity ranging from $10^{9}$ to $10^{14} \mathrm{~Pa}$.s (see Table 1) provide plausible conditions to generate a transient dynamo that may have occurred in the early history of terrestrial planets or immediately after a giant impact and the subsequent core merging process. However, the magnetic field generated by a single diapir in our models is too weak to contribute to the paleomagnetic field recorded on Mars or on the Moon.

[37] Several questions arise from our results:

[38] 1. What is the influence of the intensity of the magnetic seed field on the feasibility of a diapir driven dynamo? We have considered in this study the very weak homogeneous interplanetary magnetic seed field. However, magnetic fields are expected to be generated in planetary cores during the early evolution of planets and moons [Monteux et al., 2011a]. The presence of a stronger magnetic field during early planetary evolutions may change the flow inside the diapir, leading to faster and stronger magnetic field amplification.

[39] 2. How will multiple diapirs affect the early planetary magnetic fields? Large diapirs are not isolated events during planetary differentiations. The presence of multiple diapirs may influence the sinking dynamics of each diapir [Manga and Stone, 1993] and hence the dynamo generation.

[40] 3. Can the magnetic field of the sinking diapirs help to start the planetary dynamo? If the convective planetary dynamo is subcritical [Christensen et al., 2001], i.e. the dynamo cannot start without a strong initial field, the core 


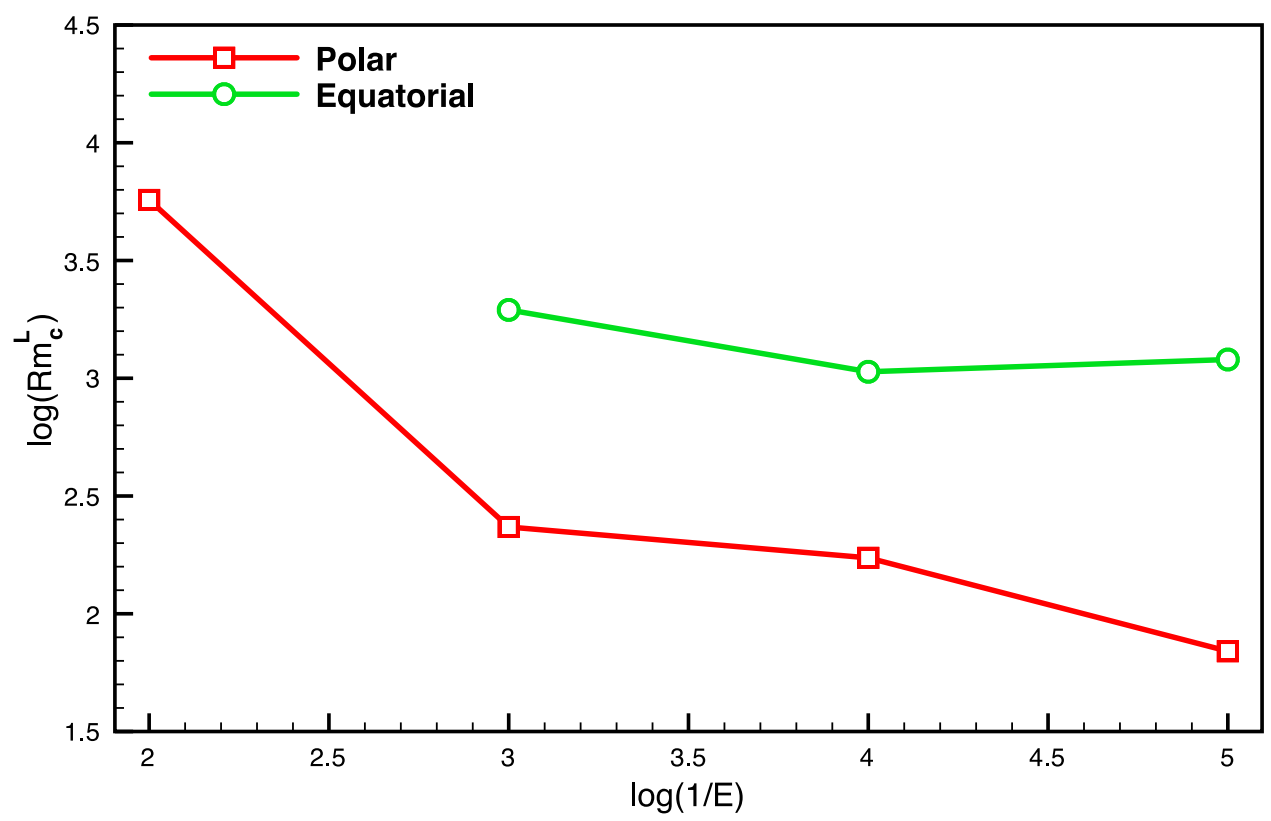

Figure 13. Local magnetic Reynolds number $R m_{c}^{L}$ as a function of $E^{-1} \cdot R m_{c}^{L}$ is obtained by combining the results for $R m_{c}$ from Figures 8 and 9 (choosing the most favorable $R o$ value for each value of $E$ ) and the fits for $h$ from Figure 5 .

merging process could bring the required magnetic field intensity inside the planetary core to trigger a dynamo.

\section{Appendix A: Numerical Method}

[41] Our three-dimensional spherical code uses a second order finite differences scheme in the radial direction and pseudo-spectral spherical harmonic expansion in the tangential direction using the high performance SHTns library (N. Schaeffer, Efficient spherical harmonic transforms aimed at pseudo-spectral numerical simulations, preprint, 2012, arXiv:1202.6522). The time stepping uses a semi-implicit Crank-Nicholson scheme for the diffusive terms, while the non-linear terms are handled by an Adams-Bashforth scheme (second order in time). To calculate the 3D flow, the momentum equation is time stepped in the spherical fluid shell with imposed velocity field at the spherical outer boundary. For kinematic dynamo simulations the induction equation is solved with an insulator outside the outer boundary and an imposed stationary 3D velocity field. The full dynamo problem can also be solved with coupled induction and momentum equations.

[42] We want to emphasize that there is no solid inner core: our code supports fluid that fills the whole sphere. The method to overcome numerical instability near $r=0$ is based on the fact that the minimum length-scale resolved by a spherical harmonic expansion truncated at degree $\ell_{\max }$ is about $\pi r / \ell_{\max }$. Hence when $r \rightarrow 0$, we resolve smaller and smaller angular length-scale, which is at best useless, and can lead to numerical instabilities. We overcome this limitation with a spherical harmonic truncation $\ell_{t r}$ that depends on $r$. Specifically, we use

$$
\ell_{t r}(r)=1+\left(\ell_{\max }-1\right) \frac{r}{r_{\max }}
$$

Although the finite difference scheme has a theoretical error that scales like $1 / r$, the numerical solutions of the velocity field and magnetic field are perfectly smooth near $r=0$, with flow and magnetic field that are allowed to cross $r=0$. We use $\ell_{\max }$ ranging from 20 to 90 and the number of radial grid points ranges from 100 to 300 depending on the strength of inertial effects (measured by the hydrodynamic Reynolds number $R e=R o / E)$.

[43] In the case of a diapir falling on the planetary pole, the boundary conditions are symmetric by rotation around the rotation axis of the planet. This implies that the stationary flow is axisymmetric (when there are no instabilities). When computing the kinematic dynamo problem with an axisymmetric flow, the various azimuthal wave numbers $m$ of the magnetic field are independent. Moreover, it is known from the Cowling theorem that the magnetic field must include non-axisymmetric $(m>1)$ terms [Cowling, 1934]. Hence we search for magnetic field with $m=1, m=2$ and $m=3$ separately. We find that most often the first growing magnetic field mode is the one with $m=2$.

[44] In the case of a diapir falling on the planetary equator, the boundary conditions are not symmetric by rotation around the rotation axis of the planet. However, they are purely $m=1$, and we find that the stationary flow, without inertial effects (small $R o / E$ ) is mainly a spiralling $m=1$ flow. When inertial effects are not negligible, all azimuthal wave numbers are present in the flow, leading to longer computations, where we usually set $m_{\max }=\ell_{\max } / 2$, which seems to be a reasonable truncation scheme when looking at the resulting spectra. The kinematic dynamo computation also requires all azimuthal wave numbers to be computed at once because they are coupled by the $m>0$ flow. We use the same truncation as for the flow, and for some cases we verified that larger truncations did not change the dynamo onset threshold significantly. 
[45] Acknowledgments. The authors thank B. Langlais and T. Seon for useful discussions. The authors also thank the anonymous reviewers for constructive comments. J.M. is funded by Agence Nationale de Recherche (Accretis decision ANR-10-PDOC-001-01). Part of the numerical simulations were run at the Service Commun de Calcul Intensif de l'Observatoire de Grenoble (SCCI).

\section{References}

Agnor, C. B., R. M. Canup, and H. F. Levison (1999), On the character and consequences of large impacts in the late stage of terrestrial planet formation, Icarus, 142, 219-237, doi:10.1006/icar.1999.6201.

Amit, H., P. Olson, and U. Christensen (2007), Tests of core flow imaging methods with numerical dynamos, Geophys. J. Int., 168, 27-39.

Batchelor, G. (1967), An Introduction to Fluid Dynamics, Cambridge Univ. Press, Cambridge, U. K.

Benz, W., W. L. Slattery, and A. G. W. Cameron (1987), The origin of the Moon and the single-impact hypothesis., Icarus, 71, 30-45, doi:10.1016/ 0019-1035(87)90160-6.

Christensen, U., and J. Aubert (2006), Scaling properties of convectiondriven dynamos in rotating spherical shells and application to planetary magnetic fields, Geophys. J. Int., 166, 97-114.

Christensen, U., and J. Wicht (2007), Numerical dynamo simulations, in Core Dynamics, Treatise Geophys., vol. 8, edited by P. Olson, pp. 245-282, Elsevier, Oxford, U. K.

Christensen, U. R., et al. (2001), A numerical dynamo benchmark, Physics Earth Planet. Inter., 128(1-4), 25-34, doi:10.1016/S0031-9201(01)00275-8

Cowling, T. (1934), The magnetic field of sun spots, Mon. Not. R. Astron. Soc., 94, 39-48.

Dahl, T. W., and D. J. Stevenson (2010), Turbulent mixing of metal and silicate during planet accretion and interpretation of the Hf-W chronometer, Earth and Planet. Sci. Lett., 295, 177-186, doi:10.1016/j.epsl.2010.03.038.

Davies, G. F. (1982), Ultimate strength of solids and formation of planetary cores, Geophys. Res. Lett., 9, 1267-1270.

Deguen, R., P. Olson, and P. Cardin (2011), Experiments on turbulent metal-silicate mixing in a magma ocean, Earth Planet. Sci. Lett., 310, 303-313, doi:10.1016/j.eps1.2011.08.041.

Dudley, M., and R. James (1989), Time-dependent kinematic dynamos with stationary flows, Proc. R. Soc. London, Ser. A, 425, 407-429.

Gailitis, A. (1970), Self-excitation of a magnetic field by a pair of ring vortices [in Russian], Mag. Gidrodin., 6, 19-22. [Magnetohydrodynamics, Engl. Transl., 6, 14-17.]

Gerya, T. V., and D. A. Yuen (2007), Robust characteristics method for modelling multiphase visco-elastic thermo-mechanical problems, Phys. Earth Planet. Inter., 163, 83-105.

Gubbins, D., C. Barber, S. Gibbons, and J. Love (2000a), Kinematic dynamo action in a sphere: I. Effects of differential rotation and meridional circulation on solutions with axial dipole symmetry, Proc. R. Soc. London, Ser. A, 456, 1333-1353.

Gubbins, D., C. Barber, S. Gibbons, and J. Love (2000b), Kinematic dynamo action in a sphere: II. Symmetry selection, Proc. R. Soc. London, Ser. A, 456, 1669-1683.

Guervilly, C., and P. Cardin (2010), Numerical simulations of dynamos generated in spherical Couette flows, Geophys. Astrophys. Fluid Dyn., 104, 221-248, doi:10.1080/03091920903550955.

Hadamard, J. (1911), Mouvement permanent lent d'une sphére liquide et visqueuse dans un liquide visqueux, C. R. Acad. Sci., 152, 1735-1738.

Höink, T., J. Schmalzl, and U. Hansen (2005), Formation of compositional structures by sedimentation in vigorous convection, Phys. Earth Planet. Inter., 153, 11-20.

Höink, T., J. Schmalzl, and U. Hansen (2006), Dynamics of metal-silicate separation in a terrestrial magma ocean, Geochem. Geophys. Geosyst. 70, Q09008, doi:10.1029/2006GC001268.

Honda, R., H. Mizutani, and T. Yamamoto (1993), Numerical simulation of Earth's core formation, J. Geophys. Res., 98, 2075-2089.

Karato, S.-I., and V. Murthy (1997), Core formation and chemical equilibrium in the Earth-I. Physical considerations, Phys. Earth Planet. Inter., $100,61-79$

Kaula, W. M. (1979), Thermal evolution of Earth and Moon growing by planetesimal impacts, J. Geophys. Res., 84, 999-1008.

Kleine, T., C. Münker, K. Mezger, and H. Palme (2002), Rapid accretion and early core formation on asteroids and the terrestrial planets from Hf-W chronometry, Nature, 418, 952-955.

Le Bars, M., M. Wieczorek, O. Karatekin, D. Cébron, and M. Laneuville (2011), An impact-driven dynamo for the early Moon, Nature, 479 215-218.

Manga, M., and H. A. Stone (1993), Buoyancy-driven interactions between two deformable viscous drops, J. Fluid Mech., 256, 647-683, doi:10.1017/S0022112093002915.
Moffatt, H. (1978), Magnetic Field Generation in Electrically Conducting Fluids, Cambridge Univ. Press, Cambridge, U. K.

Monteux, J., Y. Ricard, N. Coltice, F. Dubuffet, and M. Ulvrova (2009), A model of metal-silicate separation on growing planets, Earth Planet. Sci. Lett., 287, 353-362.

Monteux, J., A. M. Jellinek, and C. L. Johnson (2011a), Why might planets and moons have early dynamos?, Earth. Planet. Sci. Lett., 310, 349-359, doi:10.1016/j.eps1.2011.08.014.

Monteux, J., M. Jellinek, and C. L. Johnson (2011b), Core merging after the Martian giant impact, Lunar Planet. Sci. Conf., XLII, Abstract 1665.

Moss, D. (2008), Simple laminar dynamos: From two rolls to one, Geophys. Astrophys. Fluid Dyn., 102, 195-203.

Olson, P. (2007), Overview on core dynamics, in Core Dynamics, Treatise Geophys., vol. 8, edited by P. Olson, pp. 1-30, Elsevier, Oxford, U. K.

Olson, P., U. Christensen, and G. Glatzmaier (1999), Numerical modeling of the geodynamo: Mechanisms of field generation and equilibration, J. Geophys. Res., 104, 10,383-10,404.

Olson, P., I. Sumita, and J. Aurnou (2002), Diffusive magnetic images of upwelling patterns in the core, J. Geophys. Res., 107(B12), 2348, doi:10.1029/2001JB000384.

Pedlosky, J. (1987), Geophysical Fluid Dynamics, Springer, New York.

Peyrot, M., F. Plunian, and C. Normand (2007), Parametric instability of the helical dynamo, Phys. Fluids, 19, 054109, doi:10.1063/1.2734118.

Ponty, Y., and F. Plunian (2011), Transition from large-scale to small-scale dynamo, Phys. Rev. Lett., 106, 154502, doi:10.1103/PhysRevLett.106. 154502 .

Ricard, Y., O. Šrámek, and F. Dubuffet (2009), A multi-phase model of runaway core-mantle segregation in planetary embryos, Earth Planet. Sci. Lett., 284, 144-150.

Riedler, W., K. Schwingenschuh, D. Moehlmann, V. N. Oraevskii, E. Eroshenko, and J. Slavin (1989), Magnetic fields near Mars-First results, Nature, 341, 604-607, doi:10.1038/341604a0.

Rubie, D., H. Melosh, J. Reid, C. Liebske, and K. Righter (2003), Mechanisms of metal-silicate equilibration in the terrestrial magma ocean, Earth Planet. Sci. Lett., 205, 239-255.

Safronov, V. S. (1978), The heating of the Earth during its formation, Icarus, 33, 3-12, doi:10.1016/0019-1035(78)90019-2.

Samuel, H. (2012), A re-evaluation of metal diapir breakup and equilibration in terrestrial magma oceans, Earth Planet. Sci. Lett., 313, 105-114, doi:10.1016/j.epsl.2011.11.001.

Samuel, H., and P. Tackley (2008), Dynamics of core formation and equilibration by negative diapirism, Geochem. Geophys. Geosyst., 9, Q06011, doi:10.1029/2007GC001896.

Schaeffer, N., and P. Cardin (2005), Quasigeostrophic model of the instabilities of the stewartson layer in flat and depth-varying containers, Phys. Fluids, 17(10), 104111, doi:10.1063/1.2073547.

Senshu, H., K. Kuramoto, and T. Matsui (2002), Thermal evolution of a growing Mars, J. Geophys. Res., 107(12), 5118, doi:10.1029/2001JE001819.

Shannon, M. C., and C. B. Agee (1996), High pressure constraints on percolative core formation, Geophys. Res. Lett., 23, 2717-2720.

Spells, K. (1952), A study of circulation patterns within liquid drops moving through a liquid, Proc. Phys. Soc. B, 65, 541-546.

Šrámek, O., Y. Ricard, and F. Dubuffet (2010), A multiphase model of core formation, Geophys. J. Int., 181, 198-220, doi:10.1111/j.1365246X.2010.04528.x.

Stevenson, D. (1981), Models of the Earth's core, Science, 214, 611-619.

Stevenson, D. J. (2003), Planetary science: Mission to Earth's coreA modest proposal, Nature, 423(6937), 239-240, doi:10.1038/423239a.

Stewartson, K. (1957), On almost rigid rotations, J. Fluid Mech., 3(01), 17-26, doi:10.1017/S0022112057000452.

Tilgner, A. (2005), Precession driven dynamos, Phys. Fluids, 17(3), 034104, doi:10.1063/1.1852576.

Tonks, W. B., and H. J. Melosh (1992), Core formation by giant impacts, Icarus, 100, 326-346.

Tonks, W. B., and H. J. Melosh (1993), Magma ocean formation due to giant impacts, J. Geophys. Res., 98, 5319-5333.

Touboul, M., T. Kleine, B. Bourdon, H. Palme, and R. Wieler (2007), Late formation and prolonged differentiation of the Moon inferred from W isotopes in lunar metals, Nature, 450, 1206-1209.

Ulvrová, M., N. Coltice, Y. Ricard, S. Labrosse, F. Dubuffet, J. Velímský, and O. Srámek (2011), Compositional and thermal equilibration of particles, drops, and diapirs in geophysical flows, Geochem. Geophys. Geosyst. 121, Q10014, doi:10.1029/2011GC003757.

Yin, Q., S. B. Jacobsen, K. Yamashita, J. Blichert-Toft, P. Télouk, and F. Albarède (2002), A short timescale for terrestrial planet formation from Hf-W chronometry of meteorites, Nature, 418, 949-952.

Yoshino, T., M. J. Walter, and T. Katsura (2003), Core formation in planetesimals triggered by permeable flow, Nature, 422, 154-157. 